\title{
Near-4D STEM with an Orbital Angular Momentum Sorter: Advantages and Challenges
}

Enzo Rotunno ${ }^{1}$, Amir Tavabi ${ }^{2}$, Paolo Rosi $^{3}$, Alberto Roncaglia ${ }^{4}$, Robert Nijland ${ }^{5}$, Moumita Ghosh ${ }^{5}$, Peter Tiemeijer $^{5}$, Stefano Frabboni ${ }^{3}$, Rafal Dunin-Borkowski ${ }^{2}$ and Vincenzo Grillo ${ }^{1}$

${ }^{1}$ CNR - Istituto Nanoscienze, Modena, Emilia-Romagna, Italy, ${ }^{2}$ Ernst Ruska-Centre for Microscopy and Spectroscopy with Electrons, Jülich, Nordrhein-Westfalen, Germany, ${ }^{3}$ Università di Modena e Reggio Emilia, Modena, Emilia-Romagna, Italy, ${ }^{4}$ CNR-IMM, Bologna, Emilia-Romagna, Italy, ${ }^{5}$ ThermoFisher, Eindoven, Noord-Brabant, Netherlands

There is increasing interest in developing scanning transmission electron microscopy (STEM) techniques, which allow a complex set of information and potentially a full diffraction pattern to be collected at each probe position, in order to determine the phase of the electron wavefunction and to maximize the information that can be obtained at every point. Among these schemes, differential phase contrast (DPC) and integrated DPC (IDPC) imaging have shown impressive results, especially for measuring fields and imaging light elements [1][2][3].

Here, we propose a 3D, or even 4D-STEM implementation, which is connected to an orbital angular momentum (OAM) sorter [4] and provides complementary information to that available from more standard 4D-STEM techniques. An OAM sorter permits OAM and radial momentum to be measured for each scan position. It can provide edge enhancement, as well as measurements of vorticity and, in principle, full phase information [5]. We have coupled an OAM sorter to a scanning convergent probe and a system of de-scan coils, in order to be able to record position-dependent OAM spectra.

Simulations performed using custom-designed multislice code are shown in Fig. 1. They show that, by singling out an OAM channel for which $\ell=1$, an edge-enhanced image of a $Q$-shaped particle can be obtained. The edge enhancement is visible in different OAM channels in different ways. Such an imaging mode corresponds to TEM imaging with a spiral phase plate and provides superior signal-to-noise ratio for a given electron dose. Parallel recording of the $+/-1$ and 0 signals using the scheme outlined recently for spiral phase plates [4] should allow for complete phase reconstruction, similar to that available using IDPC imaging. In-depth analysis should be carried out to compare the two techniques. Figure 2 shows an example of an application of standard STEM imaging techniques and the new near-4D STEM approach to proteins. Here, the channel $\ell=1$ provides a signal that is similar to inverse bright-field imaging.

This work is supported by Q-SORT, a project funded by the European Union's Horizon 2020 Research and Innovation Program under grant agreement No. 766970. 

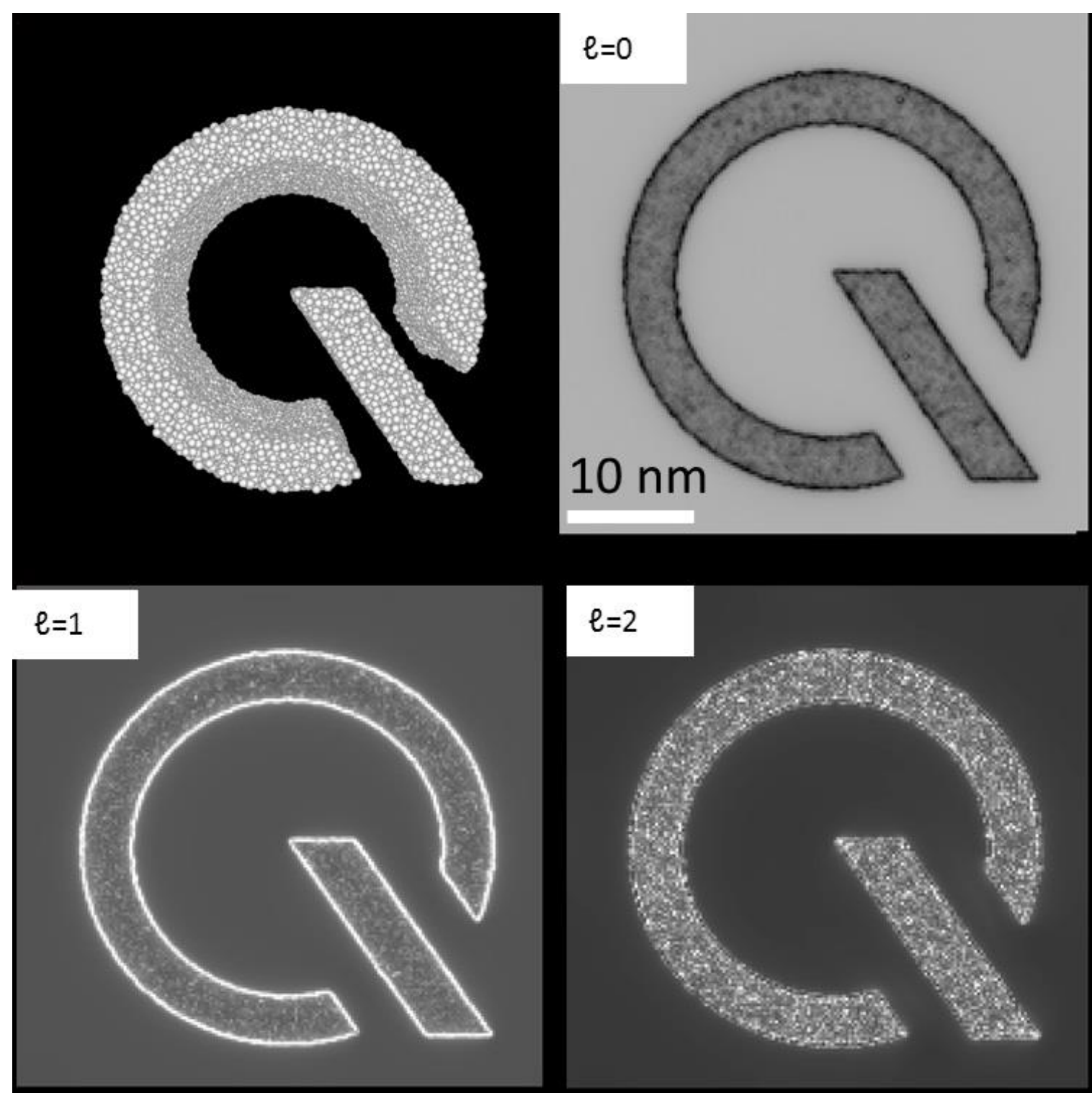

Figure 1. Simulation of a Q-shaped particle, with the atomistic model shown in the upper left frame, imaged using near-4D-STEM with an OAM sorter for channels $\ell=0,1,2$. Edge enhancement can be seen for $\ell=1$ and inversely for $\ell=0$. 

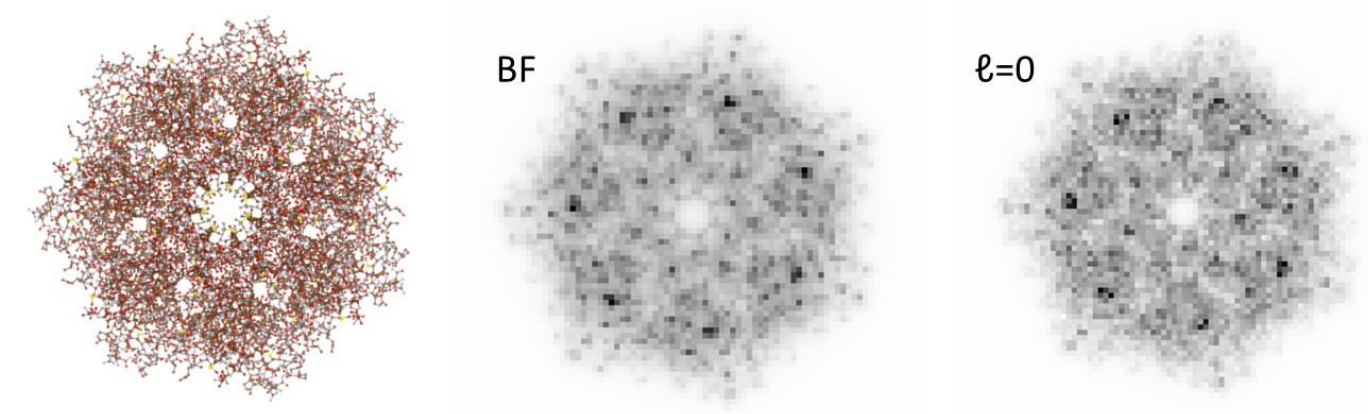

$5 \mathrm{~nm}$

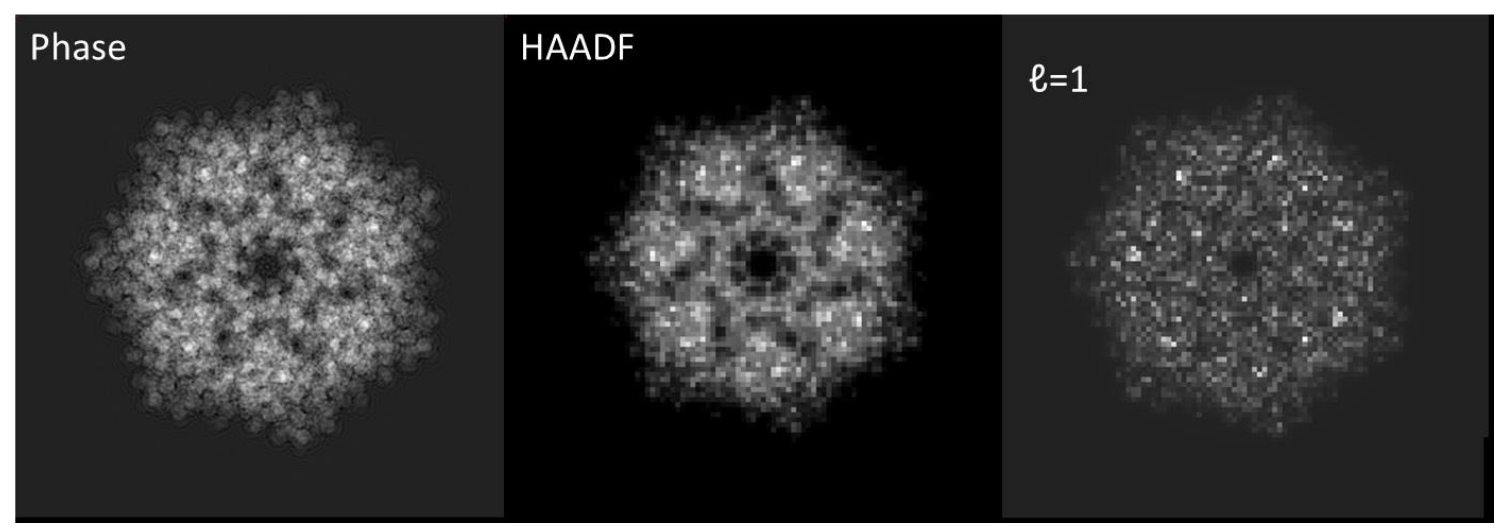

Figure 2. Simulations performed for STEM analysis of a protein using bright-field (BF) and high-angle annular dark-field (HAADF) detectors (center column) and STEM with an OAM sorter (right column) for the $\ell=0$ and $\ell=1$ channels.

\section{References}

[1] Shibata, N. et al. Nat. Phys. 8, 611-615 (2012)

[2] E. Yücelen, I. Lazić \& E. G. T. Bosch Scientific Reports 8, 2676 (2018)

[3] K Müller et al. Nature Communications volume 5, 5653 (2014)

[4] V. Grillo, A. H. Tavabi et al. Nat. Comm. 8, 15536 (2017).

[5] R Juchtmans, L. Clark Phys. Rev. A 94, 023838 (2016) 